\title{
HD-RACE: Spray-based Local Tone Mapping Operator
}

\author{
Karlo Koščević*, Vedran Stipetić*, Edoardo Provenzi ${ }^{\dagger}$, Nikola Banić $\ddagger$, Marko Subašićc, Sven Lončarić* \\ ${ }^{*}$ University of Zagreb Faculty of Electrical Engineering and Computing, Zagreb, Croatia \\ ${ }^{\dagger}$ Institute de Mathematics, Université de Bordeaux, Talence, France \\ ${ }^{\ddagger}$ Gideon Brothers, Zagreb, Croatia \\ Email: karlo.koscevic@fer.hr
}

\begin{abstract}
In this paper, a local tone mapping operator is proposed. It is based on the theory of sprays introduced in the Random Sprays Retinex algorithm, a white balance algorithm dealing with the locality of color perception. This tone mapping implementation compresses high dynamic range images by using three types of computations on sprays. These operations are carefully chosen so that the result of their convex combination is a low dynamic image with a high level of detail in all its parts regardless of the original luminance values that may span over large dynamic ranges. Furthermore, a simple local formulation of the Naka-Rushton equation based on random sprays is given. The experimental results are presented and discussed.

Index Terms-High dynamic range images, tone mapping, Naka-Rushton equation, Retinex
\end{abstract}

\section{INTRODUCTION}

High Dynamic Range (HDR) imaging is becoming a more prominent way of storing image data. It enables the capture of a much greater range of radiance values than conventional Low Dynamic Range (LDR) images, and, therefore, provides a more accurate representation of the real world. However, since devices such as displays and printers are not all capable of displaying values that span such wide ranges of magnitudes, it is necessary to reduce the dynamic range of HDR images to properly visualize them on LDR display devices. The procedure referred to as Tone Mapping (TM) is used for such conversion, and a method that performs TM is called Tone Mapping Operator (TMO). TMOs can either apply the same mapping function to all image pixels (global TMOs) or spatially variant mapping functions based on the pixel neighborhood (local TMOs).

In this paper, a local TMO combining multiple spraybased TMOs and Naka-Rushton equation [1] is proposed. The proposed TMO uses the luminance values of the input HDR image and performs three types of TM with each type producing the optimal result for a specific part of the dynamic range. With this approach, a sufficiently high level of detail can be retained in both very dark and very bright parts of an HDR image. The proposed TMO has three parameters that control the final look of the LDR image, which are highly dependent on the image content, as discussed in later sections. Therefore, the parameter tuning is performed by the user to match her/his

This work was supported by the Croatian Science Foundation under Grant DOK-01-2018. own preference. Additionally, a local formulation of the NakaRushton equation based on sprays is proposed. This ensures that all TM calculations of the proposed TMO are entirely performed on local regions represented as sprays.

\section{A. Tone mapping state-of-the-art}

TMOs can be roughly divided into global and local ones. If a global TMO is used, then the pixels that have the same intensity in the HDR image will also have the same intensity in the LDR image, i.e. all pixels with the same intensity are processed in the same way. On the other hand, for each pixel, a local TMO will during its processing take into account both its intensity and the intensities of its neighboring pixels. Global TMOs are usually faster, while local TMOs are generally considered to give results of higher perceptual quality.

Some of the earlier global TMOs are based on the imitation of human response to light [2]-[5], application of Steven's law [6]-[8], sigmoidal contrast enhancement [9], histogram adjustment [10], Naka-Rushton equation [11]. Examples of local TMOs include application of bilateral filtering of the image base layer [12], anisotropic diffusion [13], luminance gradient field manipulation [14], [15], applying the experience of photographic practice [16], relying on the Retinex theory [17]-[19], using localized applications of the NakaRushton equation [11]. More recently, deep learning has also been widely applied to the problem of TM [20]-[29].

Judging which TMO results in LDRs of higher quality is still an open question. While there are objective metrics such as Tone Mapped image Quality Index (TMQI) [30], its improved version [31], Feature Similarity Index For ToneMapped images (FSITM) [32], [33], etc., these can often be manipulated and thus they are not always reliable [34]. Because of that, various combinations of evaluations are used.

\section{ReCAll of RSR, ACE, RACE, AND NAKA-RUSHTON}

\section{A. Random spray retinex}

In [35] Land and McCann introduced Retinex, one of the first models of vision that copes with local adaptability. This locality was accomplished by using paths connecting a pixel to other pixels in the image. This sampling over many paths is computationally expensive, and so using the analysis from [36], the problem was rephrased from using paths to using sets of pixels scattered around the image. These 
sets are called random sprays, and so the method is called random spray retinex (RSR). Mathematically, let $S_{i}(x) \subset \mathbb{R}^{2}$, $1 \leq i \leq N$, be sets of $n_{i}$ pixels each centered around some pixel $x$. Then, the output value of the RSR algorithm at pixel $x$ is defined as

$$
L^{\mathrm{RSR}}(x)=\frac{1}{N} \sum_{i=1}^{N} \frac{I(x)}{\max _{y \in S_{i}(x)} I(y)} .
$$

\section{B. Automatic color equalization}

Automatic color equalization, or ACE for short, introduced in [37], is an algorithm for color enhancement inspired by some perceptual properties of vision. The process is done in two steps, the first one is the chromatic/spatial adaptation, and the second one is the dynamic tone reproduction scaling. Let $S(x)$ be some subset of pixels around a given pixel $x$, the chromatic/spatial adaptation of the pixel $x$ is defined as

$$
R(x)=\frac{\sum_{y \in S(x)} \frac{r(I(x)-I(y)}{d(x, y)}}{\sum_{y \in S(x)} \frac{r_{\max }}{d(x, y)}} .
$$

Here $d$ is a distance function, for instance Euclidean distance, $e^{-\alpha x}$ or Manhattan distance. The function $r$ can be chosen to be either a linear function, the signum function or the saturation function, that takes values -1 for $x<-\varepsilon, 1$ for $x>\varepsilon$ for some small $\varepsilon$ and connects the two parts linearly. Saturation is the most commonly used choice. In the second step, the final image is calculated using dynamic TM.

$$
L^{\mathrm{ACE}}(x)=\text { round }\left(127.5+\frac{255}{R_{\max }-R_{\min }} R(x)\right) .
$$

This way the image always stretches to fill in the entire dynamic range from 0 to 255 .

\section{Retinex automatic color equalization}

In [38] the authors combined RSR and ACE into a single method called RACE. In RACE localization is once again done by using sprays, just like in RSR, and the formula can be seen as an average of the white patch nature of retinex and the gray world nature of ACE. The corrected image is given as

$L^{\mathrm{RACE}}(x)=\frac{1}{N} \sum_{i=1}^{N} \frac{1}{2}\left(\frac{I(x)}{\max _{y \in S_{i}(x)} I(y)}+\frac{1}{n_{k}} \sum_{y \in S_{i}(x) \backslash\{x\}} r(I(x)-I(y))\right)$.

The RACE method can be seen as an improvement of both RSR and ACE since it gives better details than RSR and it prevents washing out colors as it may happen with ACE.

\section{Naka-Rushton equation}

The Naka-Rushton equation is used for modelling the adaptability of the human visual system to a large range of radiances. In [1] the authors developed an equation to model this adaptation of the human visual system, as well as confirmed it using neuroscience experiments. The equation is:

$$
r(I)=\frac{I}{I+I_{s}}
$$

where $I_{s}$ is the semi-saturation level and equal to the light intensity level at which the retinal neuron response reaches the half-maximal value. The $r$ models the neuronal response to the light.

\section{ADAPTATION TO HDR IMAGING CONDITIONS}

In previous sections, different well-established color enhancement algorithms are described. However, by introducing the proper notation and slight modifications to algorithms they can be extended for HDR imaging. The notation that will be used in the rest of the paper is defined in the following text. Let $\Omega=\{1, \ldots, W\} \times\{1, \ldots, H\} \subset \mathbb{Z}^{2}$ be the spatial domain of the input HDR image, where $W, H \geq 1$ are the image width and height, respectively, and let $\vec{I}: \Omega \rightarrow(0,+\infty)^{3}$ be the radiance map of the same HDR image. Then, $I_{c}(x)$ denotes the intensity value in the channel $c$ of the pixel $x$, where $c \in\{R, G, B\}$, and $x=\left(x_{1}, x_{2}\right) \in \Omega$. The luminance of a pixel $x \in \Omega$ is denoted with $\lambda(x)$ and is computed as:

$$
\lambda(x)=\frac{1}{3} \sum_{c \in\{R, G, B\}} I_{c}(x), \quad \forall x \in \Omega .
$$

All of the TM operations in the proposed method are based on localized sprays introduced in RSR. A spray with $n$ points centered in a pixel $x \in \Omega$ is denoted with $S(x)$. With $N$, the number of sprays per pixel is denoted.

\section{A. Local Naka-Rushton equation}

An important part in the proposed TMO is the NakaRushton equation. To comply with HDR imaging conditions, (5) has to be redefined as follows:

$$
r(x)=\frac{I(x)}{I(x)+\mu},
$$

where $\mu$ represents the semi-saturation level. According to [39], in this paper, the choice of $\mu$ is fixed to $\mu=$ $\left(\mu^{a} \cdot \mu^{g}\right)^{1 / 2}$, where $\mu^{a}$ and $\mu^{g}$ are arithmetic and geometric averages, respectively.

The modification proposed in this paper is to apply the Naka-Rushton equation to local image regions by using sprays. This means that the semi-saturation level cannot be computed from image averages but with respect to the averages of the values within the spray. Therefore, semi-saturation level $\mu$ is not a single fixed value but changes depending on the spray position and pixels contained within the spray. Local NakaRushton is then defined as:

$$
L^{\mathrm{NR}}(x, S)=\frac{I(x)}{I(x)+\mu_{S(x)}},
$$

where $S(x)$ denotes a spray centered in a pixel $x$, and $\mu_{S(x)}=$ $\left(\mu_{S(x)}^{a} \cdot \mu_{S(x)}^{g}\right)^{1 / 2}$ with $\mu_{S(x)}^{a}$ and $\mu_{S(x)}^{g}$ being the arithmetic and geometric averages of the spray, respectively 


\section{B. Naka-Rushton RSR}

An improvement of both RSR and Naka-Rushton as TMOs can be achieved by the fusion of the two together. In the simplest form, this fusion can be achieved by first applying (5) on an HDR image to compress the range of radiance values and then applying (1). However, such an approach cannot be considered locally adaptive since the compression performed by the Naka-Rushton equation is based on the arithmetic and geometric averages of the whole image. Therefore, in this paper, local Naka-Rushton defined by (8) is used to perform the fusion and model the local adaptability. In other words, before dividing some pixel $x$ with the maximum in the spray which is centered in $x$ (as it is done in RSR) local NakaRushton is computed on the spray including the pixel $x$. As in RSR, for each $x \in \Omega$ the results of $N$ sprays are averaged. Formally, the fusion of RSR and Naka-Rushton is defined as

$$
L^{\mathrm{NR}-\mathrm{RSR}}(x)=\frac{1}{N} \sum_{i=1}^{N} \frac{L^{\mathrm{NR}}\left(x, S_{i}\right)}{\max _{y \in S_{i}(x)} L^{\mathrm{NR}}\left(y, S_{i}\right)}
$$

\section{ACE Adaptation}

In this paper, similar to [38], ACE is fused with RSR and due to the local white patch behaviour of RSR the dynamic tone reproduction in ACE is removed. The slope function is then computed as

$$
s_{\alpha_{S(x)}}(\Delta)= \begin{cases}0 & \Delta \leq-\frac{1}{2 \alpha_{S(x)}} \\ \frac{1}{2}+\alpha_{S(x)} \Delta & |\Delta|<\frac{1}{2 \alpha_{S(x)}} \\ 1 & \Delta \geq \frac{1}{2 \alpha_{S(x)}}\end{cases}
$$

where $\alpha_{S(x)} \geq 1, \forall x \in S(x)$ is a local pixel-dependent slope built as follows:

$$
\alpha_{S(x)}=\frac{\max _{y \in S(x)} I(y)}{\min _{y \in S(x)} I(y)} .
$$

With this choice $\alpha_{S(x)}$ is illuminant-independent, i.e. independent under the scaling by a positive constant representing the color of a global and uniform illuminant, and it will be big in areas covered by the spray in which the variation between the minimal and the maximal intensities is large. This will allow a very efficient detail rendition of this kind of area, which is well-known to be particularly difficult to tone-map.

For the given definition of the slope function and the slope, the image range must be contained in $[0,1]$. Therefore, instead of applying ACE on the pure values of $\vec{I}$, just like in NakaRushton RSR (III-B), local Naka-Rushton is first applied on $S(x)$. Denoting the resulting intensity of the center pixel as $\tilde{I}(x)$ and the resulting intensity of any other pixel in a spray as $\tilde{I}(y)$ the formula for NR-ACE is

$$
L^{\mathrm{NR}-\mathrm{ACE}}(x)=\frac{1}{N} \sum_{i=1}^{N} \frac{1}{\tilde{n}_{i}} \sum_{y \in S_{i}(x) \backslash\{x\}} s_{\alpha_{S_{i}(x)}}(\tilde{I}(x)-\tilde{I}(y)),
$$

where $\tilde{n}_{i}$ is the number of pixels in the $i$ th spray excluding the center pixel.

\section{Algorithm DESCRIPTION AND RESUlts}

In this section, the fusion of RSR, NR-RSR, and NR-ACE is described. Each of these TMOs produces a different-looking LDR version of the same image with the following distinct features: RSR can work well in very bright parts, NR-ACE covers very dark parts, and NR-RSR works as an intermediate between the two. When combined, outputs of these TMOs can give very pleasant LDR images in both color and detail. In this paper, they are combined locally in a pixel-wise convex combination by utilizing the random sprays technique.

All of the TM operations are performed based on the luminance values of an image. Therefore, (6) is first applied to the input HDR image to compute the luminance for each pixel. The luminance is then normalized to have the minimum in zero as follows:

$$
\lambda_{0}(x)=\lambda(x)-\min _{y \in \Omega} \lambda(y)
$$

Normalized luminance is tone mapped in three different ways by applying (1), (9), and (12).

To avoid having the pixels of a spray fall outside the bounds of an image when extracting the sprays, images are expanded by mirroring them in the vertical, horizontal, and diagonal direction, as shown in Fig. 1.

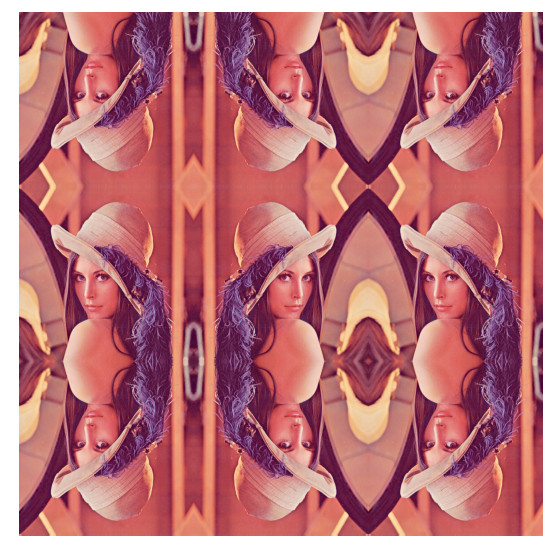

Fig. 1: The example of expanding an image by mirroring in vertical, horizontal and diagonal direction.

The final tone mapped luminance is computed in two steps. Frist, the output of pure RSR ( $L^{\mathrm{RSR}}$ ) and Naka-Rushton RSR $\left(L^{\mathrm{NR}-\mathrm{RSR}}\right)$ are fused as follows:

$$
L^{\mathrm{HD}-\mathrm{RSR}}(x)=\beta(x) L^{\mathrm{RSR}}(x)+(1-\beta(x)) L^{\mathrm{NR}-\mathrm{RSR}}(x),
$$

where $\beta(x)$ is a per-pixel parameter controlling the amount of RSR and NR-RSR in each pixel. Similar, in the second step, Naka-Rushton ACE $\left(L^{\mathrm{NR}-\mathrm{ACE}}\right)$ is fused in with $L^{\mathrm{HD}-\mathrm{RSR}}$ as follows:

$$
L^{\mathrm{HD}-\mathrm{RACE}}(x)=\gamma(x) L^{\mathrm{NR}-\mathrm{ACE}}(x)+(1-\gamma(x)) L^{\mathrm{HD}-\mathrm{RSR}}(x),
$$

where $L^{\mathrm{HD}-\mathrm{RACE}}$ is the final tone mapped luminance and $\gamma(x)$ the regulating factor. 
The next step is to extend $L^{\mathrm{HD}-\mathrm{RACE}}$ to color images. In [40], TM operations are extended to color images by applying the modified Naka-Rushton formula as follows:

$$
r(x)=\frac{I_{c}(x)}{I_{c}(x)+f_{\mu}(\lambda(x))},
$$

where $f_{\mu}(\lambda(x))$ is a function the authors introduce to ensure that Naka-Rushton equation follows the Weber-Fechner law. Based on (16), in this paper, the extension to color images is

$$
L_{c}(x)=L(x) \frac{I_{c}(x)}{L(x) I_{c}(x)+(1-L(x)) \lambda(x)} .
$$

Here, for each color channel $c \in\{R, G, B\}$, the denominator is the convex combination between the intensity of some pixel $x \in \Omega$ in that channel $I_{c}(x)$ and the luminance of the same pixel $\lambda(x)$ based on the $L(x) . L(x)$ is the tone mapped luminance having the values in range $[0,1]$. The proposed conversion ensures that the following properties are satisfied:

- when $L(x)=0$ it is true that $L_{c}(x)=0$,

- when $L(x)=1$ it is true that $L_{c}(x)=1$,

- and when $L(x)=\frac{1}{2}$ pixel intensity in a color channel and pixel luminance are equally valued, i.e., that $L_{c}(x)=$ $I_{c}(x) /\left(I_{c}(x)+\lambda(x)\right)$,

By substituting $L(x)$ in (17) with $L^{\mathrm{HD}-\mathrm{RACE}}(x)$, the output of the proposed method is acquired. The pseudocode of the proposed TMO is given in Algorithm 1. In Fig. 2, a few examples of applying the proposed TMO are shown ${ }^{1}$.

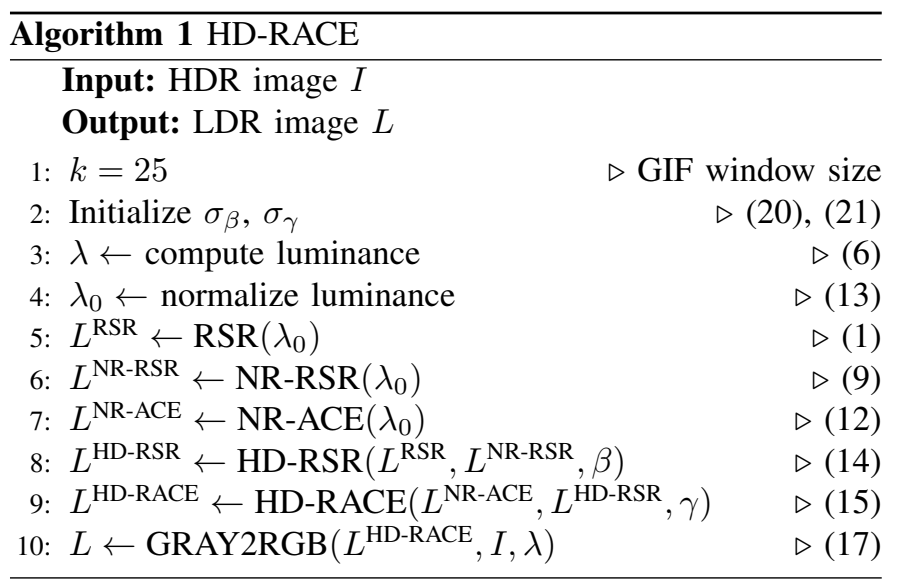

\section{A. Determining convex combination coefficients}

The coefficients $\beta(x)$ and $\gamma(x)$ tune the contribution of each version of the tone-mapped input image to the final result. The coefficient $\beta$ regulates the convex combination of RSR and NR-RSR in the final image whereas the coefficient $\gamma$ tunes the amount of NR-ACE that should be added to that combination. Both coefficients are local and derived from the luminance of each image pixel. They are computed as Gaussians with

\footnotetext{
${ }^{1}$ For all images, the number of sprays was 15 , and the number of points per spray was 250
}

respect to the zero-minimum log-luminance ${ }^{2} l$ of each image pixel as follows:

$$
\begin{gathered}
\beta(x)=e^{-\frac{\left(l(x)-\mu_{\beta}\right)^{2}}{2 \sigma_{\beta}^{2}},}, \\
\gamma(x)=1-e^{-\frac{\left(l(x)-\mu_{\gamma}\right)^{2}}{2 \sigma_{\gamma}^{2}}},
\end{gathered}
$$

where $\mu_{\beta}$ and $\mu_{\gamma}$ are equal to the maximum and minimum of $l$, respectively. $\sigma_{\beta}$ and $\sigma_{\gamma}$ are image dependent-parameters and can be adjusted according to user preference. In this paper, they are fixed to the following:

$$
\begin{gathered}
\sigma_{\beta}=0.5 \cdot\left(\sigma(l)+\max _{x} l(x)-\mathrm{Q}_{3}(l)\right), \\
\sigma_{\gamma}=0.5 \cdot\left(\sigma(l)+\mathrm{Q}_{1}(l)\right),
\end{gathered}
$$

where $Q_{1}$ and $Q_{3}$ denote the first and third quartile, respectively. These formulas are experimentally determined as a unique solution yielding satisfactory results for all test images subjectively judged by the authors. However, by tweaking these parameters even better results can be achieved but it has to be done for each individual image. Since $l$ is normalized to have the minimum in zero it means that $\mu_{\gamma}=0 . \beta(x)=1$ for all pixels of position $x$ such that $l(x)=\mu_{\beta}$ and it will decrease to 0 for all pixels with lower luminance. The decreasing speed is tuned by the value of $\sigma_{\beta}$. The similar applies to $\gamma(x)$ and its parameters $\mu_{\gamma}$ and $\sigma_{\gamma}$. However, $\beta(x)$ is defined to be equal to 1 for pixels of position $x$ corresponding to the regions in the brightest parts on an image, and for $\gamma$ it is the opposite, i.e. $\gamma$ is equal to 1 in the darkest parts of an image. This corresponds to the observations that RSR produces the most pleasing colors in the brightest image parts, and the observations that NR-ACE produces the most pleasing colors in the darkest image parts. NR-RSR is used to tune in the intermediate parts.

Guided image filtering (GIF) [41] is further applied to coefficients $\beta$ and $\gamma$. It ensures that $\beta$ and $\gamma$ are smooth in the uniform regions and preserves the changes on the edges. Consequently, the transitions between fused operators are more prominent which makes tiny details in an image more vivid and removes outliers in the regions where one operator should be dominant. The example of applying the proposed TMO with and without GIF is shown in Fig. 3. The experimental results in this paper are obtained with GIF parameters fixed to: window size $k=25$, guidance image was the input image, and regularization $\epsilon$ was equal to the variance of the input image (input image was either $\beta$ or $\gamma$ ).

\section{Discussion}

Here we discuss the limitations of the proposed TMO, which is shown by experimental results to produce pleasant results by combining key features from the three base images $L^{\mathrm{RSR}}, L^{\mathrm{NR}-\mathrm{RSR}}$, and $L^{\mathrm{NR}-\mathrm{ACE}}$, each of which shows good details in different parts of the image: RSR in the very bright parts, NR-RSR in the intermediate parts, and NR-ACE in the

\footnotetext{
${ }^{2} l$ is computed from $\lambda(x)$ as follows: 1) zeros are clipped to the smallest positive non-zero value of $\lambda(x) 2$ ) the logarithm with base 10 is computed, 3 ) minimum is subtracted from all values to center the minimum of $l$ in zero.
} 

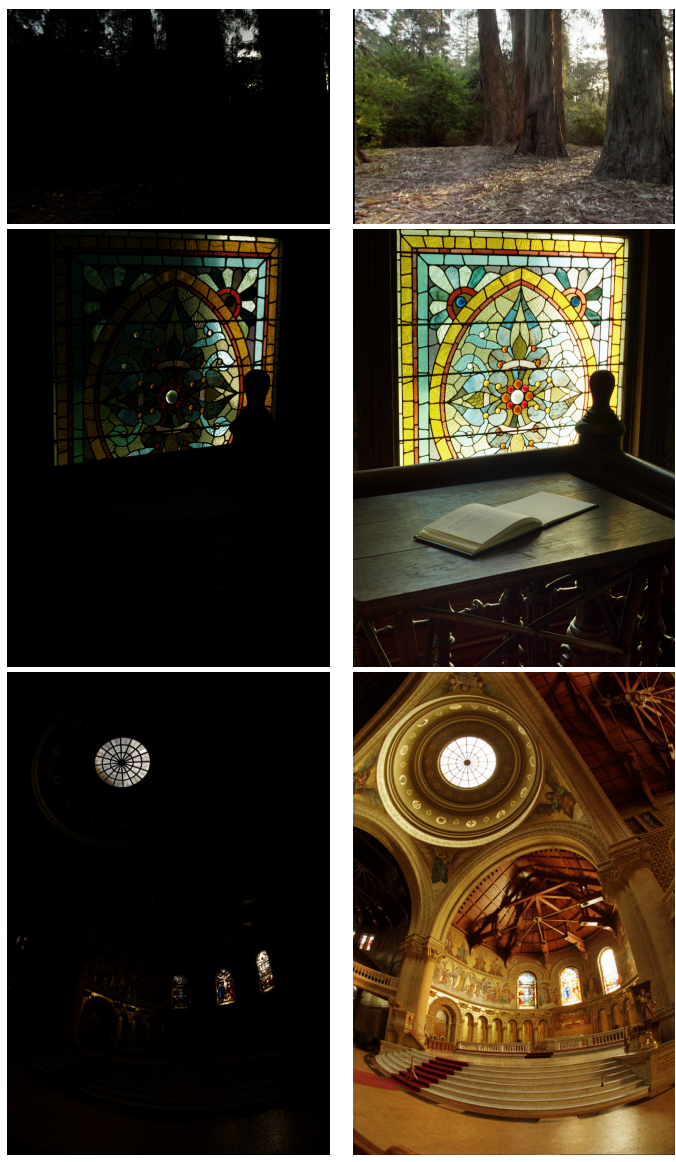
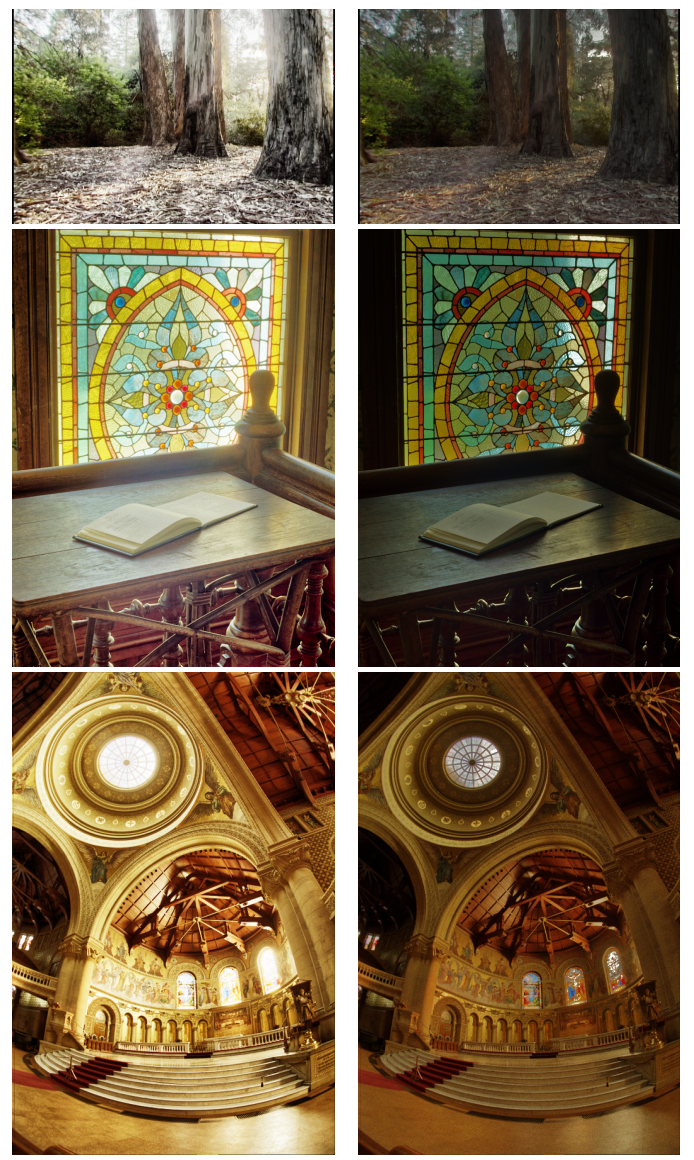

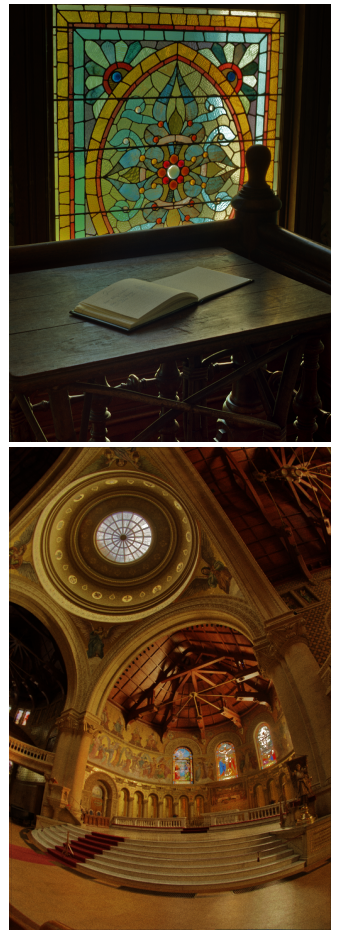

Fig. 2: Examples of applying the proposed TMO to HDR images. From left, columns are outputs of: $L^{\mathrm{RSR}}, L^{\mathrm{NR}-\mathrm{RSR}}, L^{\mathrm{NR}-\mathrm{ACE}}$, $L^{\mathrm{HD}-\mathrm{RSR}}$, and $L^{\mathrm{HD}-\mathrm{RACE}}$. Color images are acquired by applying (17) with $L$ being substituted considering the given column.

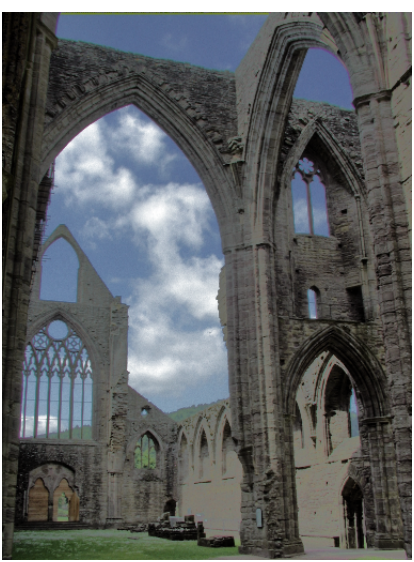

(a)

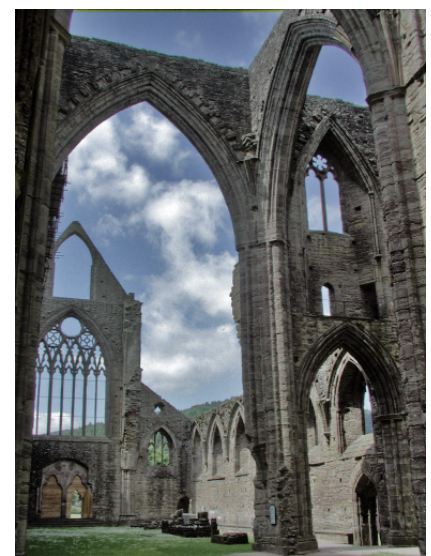

(b)
Fig. 3: The difference between an output LDR image with GIF omitted (a) and GIF applied (b) when computing $\beta$ and $\gamma$.

darker parts. This can be seen nicely in Fig. 2. However, the proposed TMO is limited by the presence of several parameters. The results in Fig. 2 are obtained by fixing the parameters to some static values which produced pleasant results for the majority of test images but more pleasing results can be achieved by tweaking the parameters. However, the optimal parameters change drastically with the image content. Therefore, in the current version of the proposed TMO, the optimal set of parameters is not given and a mechanism for automatic determination of the GIF window size and $\sigma_{\beta}$ and $\sigma_{\gamma}$ parameters from the image is yet to be explored.

The second limitation of the proposed TMO in its current form is the execution time. The majority of computations in the proposed TMO are related to spray computations for each pixel, which makes the TMO slow to execute. However, since the proposed TMO is based on the RSR, the number of computations can be reduced by incorporating the improvements of the basic RSR introduced in [42], [43].

\section{CONCLUSION}

A new tone mapping operator based on a local fusion of well-known TMOs, random spray retinex, automatic color equalization, and Naka-Rushton's equation, is proposed. The fusion is done using convex combinations of these operators with the coefficients chosen so that each operator is given more weight in areas where it gives good information. RSR in the 
very bright parts of the image, NR-RSR in the intermediate parts, and NR-ACE in the darker parts. The main drawbacks of the proposed operator are its speed and the presence of several image-dependent parameters. A method to automatically determine these parameters is proposed, a method for choosing them optimally is left for further study.

\section{REFERENCES}

[1] R. Shapley and C. Enroth-Cugell, "Visual adaptation and retinal gain controls," Progress in retinal research, vol. 3, pp. 263-346, 1984.

[2] C. Schlick, "Quantization techniques for visualization of high dynamic range pictures," in Photorealistic Rendering Techniques. Springer, 1995, pp. $7-20$.

[3] S. N. Pattanaik, J. Tumblin, H. Yee, and D. P. Greenberg, "Timedependent visual adaptation for fast realistic image display," in Proceedings of the 27th annual conference on Computer graphics and interactive techniques. ACM Press/Addison-Wesley Publishing Co., 2000, pp. 4754.

[4] F. Drago, K. Myszkowski, T. Annen, and N. Chiba, "Adaptive logarithmic mapping for displaying high contrast scenes," in Computer Graphics Forum, vol. 22, no. 3. Wiley Online Library, 2003, pp. 419-426.

[5] E. Reinhard and K. Devlin, "Dynamic range reduction inspired by photoreceptor physiology," Visualization and Computer Graphics, IEEE Transactions on, vol. 11, no. 1, pp. 13-24, 2005.

[6] J. Tumblin and H. Rushmeier, "Tone reproduction for realistic images," Computer Graphics and Applications, IEEE, vol. 13, no. 6, pp. 42-48, 1993.

[7] K. Chiu, M. Herf, P. Shirley, S. Swamy, C. Wang, K. Zimmerman et al., "Spatially nonuniform scaling functions for high contrast images," in Graphics Interface. CANADIAN INFORMATION PROCESSING SOCIETY, 1993, pp. 245-245.

[8] G. Ward, "A contrast-based scalefactor for luminance display," Graphics gems IV, pp. 415-421, 1994

[9] G. J. Braun and M. D. Fairchild, "Image lightness rescaling using sigmoidal contrast enhancement functions," Journal of Electronic Imaging, vol. 8, no. 4, pp. 380-393, 1999 .

[10] G. W. Larson, H. Rushmeier, and C. Piatko, "A visibility matching tone reproduction operator for high dynamic range scenes," Visualization and Computer Graphics, IEEE Transactions on, vol. 3, no. 4, pp. 291-306, 1997.

[11] N. Banić and S. Lončarić, "Flash, Storm, and Mistral: HardwareFriendly and High Quality Tone Mapping," in International Joint Conference on Computer Vision, Imaging and Computer Graphics. Springer, 2018, pp. 228-242.

[12] F. Durand and J. Dorsey, "Fast bilateral filtering for the display of highdynamic-range images," ACM transactions on graphics (TOG), vol. 21 no. 3, pp. 257-266, 2002

[13] J. Tumblin and G. Turk, "LCIS: A boundary hierarchy for detailpreserving contrast reduction," in Proceedings of the 26th annual conference on Computer graphics and interactive techniques. ACM Press/Addison-Wesley Publishing Co., 1999, pp. 83-90.

[14] R. Fattal, D. Lischinski, and M. Werman, "Gradient domain high dynamic range compression," in ACM Transactions on Graphics (TOG), vol. 21 , no. 3. ACM, 2002, pp. 249-256.

[15] R. Mantiuk, K. Myszkowski, and H.-P. Seidel, "A perceptual framework for contrast processing of high dynamic range images," ACM Transactions on Applied Perception (TAP), vol. 3, no. 3, pp. 286-308, 2006.

[16] E. Reinhard, M. Stark, P. Shirley, and J. Ferwerda, "Photographic tone reproduction for digital images," in ACM Transactions on Graphics (TOG), vol. 21, no. 3. ACM, 2002, pp. 267-276.

[17] L. Meylan and S. Susstrunk, "High dynamic range image rendering with a retinex-based adaptive filter," Image Processing, IEEE Transactions on, vol. 15 , no. 9 , pp. $2820-2830,2006$.

[18] N. Banić and S. Lončarić, "Color Badger: A Novel Retinex-Based Local Tone Mapping Operator," in Image and Signal Processing. Springer, 2014, pp. 400-408.

[19] _- "Puma: A High-Quality Retinex-Based Tone Mapping Operator," in Signal Processing Conference (EUSIPCO), 2016 24rd European. IEEE, 2016, pp. 943-947.

[20] X. Hou, J. Duan, and G. Qiu, "Deep Feature Consistent Deep Image Transformations: Downscaling, decolorization and HDR Tone Mapping," arXiv preprint arXiv:1707.09482, 2017.
[21] V. A. Patel, P. Shah, and S. Raman, "A Generative Adversarial Network for Tone Mapping HDR Images," in National Conference on Computer Vision, Pattern Recognition, Image Processing, and Graphics. Springer, 2017, pp. 220-231.

[22] A. Rana, G. Valenzise, and F. Dufaux, "Learning-Based Tone Mapping Operator for Efficient Image Matching," IEEE Transactions on Multimedia, vol. 21, no. 1, pp. 256-268, 2018.

[23] Q. Yan, D. Gong, Q. Shi, A. v. d. Hengel, C. Shen, I. Reid, and Y. Zhang, "Attention-Guided Network for Ghost-Free High Dynamic Range Imaging," in Proceedings of the IEEE/CVF Conference on Computer Vision and Pattern Recognition, 2019, pp. 1751-1760.

[24] A. Ignatov, L. Van Gool, and R. Timofte, "Replacing Mobile Camera ISP with a Single Deep Learning Model," in Proceedings of the IEEE/CVF Conference on Computer Vision and Pattern Recognition Workshops, 2020, pp. 536-537.

[25] Q. Yan, L. Zhang, Y. Liu, Y. Zhu, J. Sun, Q. Shi, and Y. Zhang, "Deep HDR Imaging via A Non-Local Network," IEEE Transactions on Image Processing, vol. 29, pp. 4308-4322, 2020.

[26] Q. Yan, B. Wang, L. Zhang, J. Zhang, Z. You, Q. Shi, and Y. Zhang, "Towards accurate HDR imaging with learning generator constraints," Neurocomputing, vol. 428, pp. 79-91, 2021.

[27] R. Montulet, A. Briassouli, and N. Maastricht, "Deep Learning for Robust end-to-end Tone Mapping." in BMVC, 2019, p. 194.

[28] A. Rana, P. Singh, G. Valenzise, F. Dufaux, N. Komodakis, and A. Smolic, "Deep Tone Mapping Operator for High Dynamic Range Images," IEEE Transactions on Image Processing, vol. 29, pp. 1285$1298,2019$.

[29] K. Panetta, L. Kezebou, V. Oludare, S. Agaian, and Z. Xia, "TMONet: A Parameter-Free Tone Mapping Operator Using Generative Adversarial Network, and Performance Benchmarking on Large Scale HDR Dataset," IEEE Access, vol. 9, pp. 39500-39517, 2021.

[30] H. Yeganeh and W. Zhou, "Objective Quality Assessment of Tone Mapped Images," Image Processing, IEEE Transactions on, vol. 22, no. 2, pp. 657-667, 2013.

[31] K. Ma, H. Yeganeh, K. Zeng, and Z. Wang, "High dynamic range image compression by optimizing tone mapped image quality index," Image Processing, IEEE Transactions on, vol. 24, no. 10, pp. 3086-3097, 2015.

[32] H. Ziaei Nafchi, A. Shahkolaei, R. Farrahi Moghaddam, and M. Cheriet, "FSITM: A Feature Similarity Index For Tone-Mapped Images," Signal Processing Letters, IEEE, vol. 22, no. 8, pp. 1026-1029, 2015.

[33] M. R. Faraji, "Full-reference tone-mapped images quality assessment," IET Image Processing, vol. 15, no. 3, pp. 763-773, 2021.

[34] N. Banić and S. Lončarić, "Sensitivity of Tone Mapped Image Quality Metrics to Perceptually Hardly Noticeable Differences," in Proceedings of The Fifth Croatian Computer Vision Workshop (CCVW 2013), no. 1. University of Zagreb Faculty of Electrical Engineering and Computing, Oct. 2016, pp. 15-18.

[35] E. H. Land and J. J. McCann, "Lightness and retinex theory," Josa, vol. 61 , no. 1, pp. 1-11, 1971.

[36] E. Provenzi, M. Fierro, A. Rizzi, L. De Carli, D. Gadia, and D. Marini, "Random spray retinex: a new retinex implementation to investigate the local properties of the model," IEEE Transactions on Image Processing, vol. 16 , no. 1 , pp. 162-171, 2006.

[37] A. Rizzi, C. Gatta, and D. Marini, "A new algorithm for unsupervised global and local color correction," Pattern Recognition Letters, vol. 24, no. 11, pp. 1663-1677, 2003.

[38] E. Provenzi, C. Gatta, M. Fierro, and A. Rizzi, "A spatially variant white-patch and gray-world method for color image enhancement driven by local contrast," IEEE transactions on pattern analysis and machine intelligence, vol. 30 , no. 10, pp. 1757-1770, 2008.

[39] S. Ferradans, M. Bertalmio, E. Provenzi, and V. Caselles, "An analysis of visual adaptation and contrast perception for tone mapping," IEEE Transactions on Pattern Analysis and Machine Intelligence, vol. 33, no. 10, pp. 2002-2012, 2011.

[40] S. Ferradans, E. Provenzi, M. Bertalmio, and V. Caselles, "Tstm: A two-stage tone mapper combining visual adaptation and local contrast enhancement," 2009.

[41] K. He, J. Sun, and X. Tang, "Guided image filtering," in European conference on computer vision. Springer, 2010, pp. 1-14.

[42] N. Banić and S. Lončarić, "Light random sprays retinex: exploiting the noisy illumination estimation," IEEE Signal Processing Letters, vol. 20, no. 12, pp. 1240-1243, 2013.

[43] _ - "Smart light random memory sprays retinex: a fast retinex implementation for high-quality brightness adjustment and color correction," JOSA A, vol. 32, no. 11, pp. 2136-2147, 2015. 